\title{
Effects of Levosimendan Preconditioning on Left Ventricular Remodeling after Myocardial Reperfusion in Acute Myocardial Infarction Patients Receiving Percutaneous Coronary Intervention
}

\author{
Shengqin Yu, Jindong Zhang \\ Heart Center, Affiliated Zhongshan Hospital of Dalian University, Dalian, China
}

\section{ABSTRACT}

Objective: Levosimendan is a novel drug often used to treat heart failure. We aimed to explore the effects of levosimendan preconditioning on left ventricular remodeling (LVR) after myocardial reperfusion in acute myocardial infarction (AMI) patients receiving percutaneous coronary intervention (PCI).

Methods: A total of 258 AMI patients treated from January 2018 to September 2020 were randomly divided into control and observation groups. Based on conventional drug therapy, levosimendan was given $30 \mathrm{~min}$ before PCI for the observation group, and dobutamine was intravenously injected for the control group. Baseline data, thrombolysis in myocardial infarction (TIMI) blood flow grade, myocardial injury markers, and LVR indices were compared, and the influencing factors for LVR were analyzed.

Results: After treatment, various degrees of blood perfusion were found, and the TIMI grade was better than that before treatment in both groups $(P<.05)$. The levels of aspartate aminotransferase, creatine kinase- $\mathrm{MB}$, cardiac troponin $\mathrm{T}$, and brain natriuretic peptide (BNP) declined in both groups, more significantly in the observation group $(P<.05)$. Left ventricular end-systolic diameter, left ventricular enddiastolic diameter, and left ventricular end-diastolic volume declined, whereas left ventricular ejection fraction rose in both groups, more significantly in the observation group $(P<$ $.05)$. Age and BNP were risk factors for LVR, whereas levosimendan preconditioning was a protective factor $(P<.05)$.

Conclusion: Levosimendan preconditioning can protect cardiac function and promote the recovery of left ventricular structure. Age and BNP are risk factors for LVR after myocardial reperfusion in AMI patients undergoing PCI, and levosimendan preconditioning is a protective factor.

\section{INTRODUCTION}

As one of the common types of coronary heart disease, acute myocardial infarction (AMI) refers to myocardial necrosis due to severe ischemia at the site of acute occlusion of coronary arteries and interruption of blood flow. Currently, the most

Received Augsut 24, 2021; accepted September 28, 2021.

Correspondence: Shengqin Yu (e-mail: lthe6232wdm@163.com). rapid and effective treatment method is percutaneous coronary intervention (PCI), which recanalizes the infarcted artery, restores blood flow, and reduces the MI area using catheter techniques. However, reperfusion injury may be caused by the rapid recovery of myocardial blood supply, leading to ventricular remodeling and affecting cardiac function [Elliott 2017].

Levosimendan, a novel drug, is often used in the treatment of heart failure. As long as heart rate and myocardial oxygen consumption are not changed significantly, levosimendan can stabilize the spatial configuration of myocardial fibrin by directly binding to troponin, thereby enhancing the myocardial contractility induced by calcium ions; thus it has a calciumsensitizing effect. Moreover, it dilates blood vessels by activating adenosine triphosphate-sensitive potassium channels, thus effectively alleviating the clinical symptoms of heart failure. In addition, it raises the concentration of cyclic adenosine monophosphate (cAMP) in myocardial cells by inhibiting phosphodiesterase, thereby exerting an additional positive inotropic effect [Mehta 2017; Agostoni 2019; Zhou 2019].

The effect of levosimendan preconditioning on left ventricular remodeling (LVR) after myocardial reperfusion in AMI patients undergoing PCI has rarely been reported. In the present study, therefore, levosimendan preconditioning was given to AMI patients before PCI, and the occurrence of LVR after myocardial reperfusion was explored to provide a basis for clinical treatment.

\section{MATERIALS AND METHODS}

\section{Subjects}

A total of 258 AMI patients treated in our hospital from January 2018 to September 2020 were selected and divided into a control group $(n=129)$ and an observation group $(n=$ 129) using a random number table. Inclusion criteria were (1) patients meeting the diagnostic criteria in the Guidelines for the Diagnosis and Treatment of Acute Myocardial Infarction [Chinese Society of Cardiology of Chinese Medical Association 2015], (2) patients $>18$ years old, (3) patients admitted within $6 \mathrm{~h}$ after the first onset of disease, (4) patients with left ventricular ejection fraction (LVEF) $<40 \%$ confirmed by echocardiography and definite left ventricular systolic dysfunction, (5) patients with indications for emergency PCI, (6) patients with complete clinical data, and (7) patients who (or whose families) signed informed consent. Exclusion criteria 
Table 1. Baseline clinical data

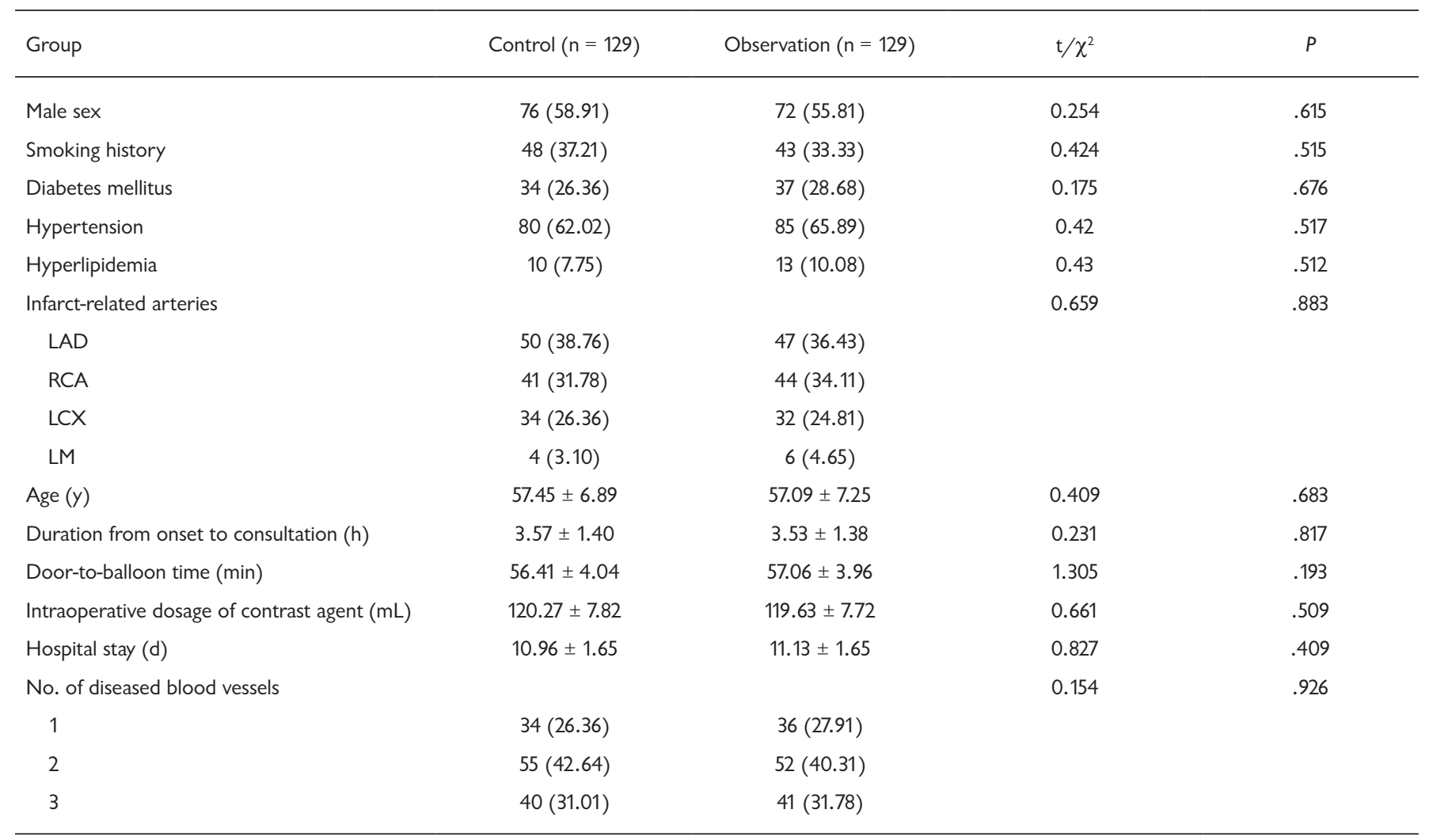

Data are $\mathrm{n}(\%)$ or mean $\pm \mathrm{SD}$. LAD, left anterior descending; LCX, left circumflex branch; LM, left main coronary artery; RCA, right coronary artery.

Table 2. TIMI grades

\begin{tabular}{|c|c|c|c|c|c|c|}
\hline Group & Grade 0 & Grade 1 & Grade 2 & Grade 1 & Grade 2 & Grade 3 \\
\hline Observation $(n=129)$ & $65(50.39)$ & $41(31.78)$ & $23(17.83)$ & $1(0.78)$ & $8(6.20)$ & $120(93.02)$ \\
\hline \multicolumn{7}{|l|}{$\chi^{2} / P$} \\
\hline Between 2 groups after $\mathrm{PCl}$ & $0.409 / 0.815$ & & & & & \\
\hline Control group & $141.497 / 0.000$ & & & & & \\
\hline Observation group & $150.082 / 0.000$ & & & & & \\
\hline
\end{tabular}

Data are $\mathrm{n}(\%)$ unless noted otherwise. PCl, percutaneous coronary intervention; TIMI, thrombolysis in myocardial infarction.

were (1) patients with severe valvular disease, pericardial disease, arrhythmia, restrictive or hypertrophic cardiomyopathy, or uncontrolled thyroid disease; (2) patients with malignant tumors, severe insufficiency of other organs, metabolic disease, immune disease, infectious disease, or coagulation dysfunction; (3) patients with a history of cardiac surgery; (4) patients who used levosimendan recently; (5) patients with contraindications to the drugs used in this study; (6) patients with emerging congestive heart failure or all-cause deaths at 3 months of follow-up; or (7) patients who quit midway or were lost to follow-up. This study was reviewed and approved by the Medical Ethics Committee of our hospital.

\section{Treatment Methods}

After the patient was admitted to the hospital, the green channel was activated immediately, and PCI was performed 
Table 3. Myocardial injury markers

\begin{tabular}{|c|c|c|c|c|}
\hline \multicolumn{5}{|l|}{ Control $(n=129)$} \\
\hline After $\mathrm{PCl}$ & $29.54 \pm 3.82$ & $10.49 \pm 1.56$ & $2.13 \pm 0.29$ & $95.42 \pm 6.23$ \\
\hline \multicolumn{5}{|l|}{ Observation $(n=129)$} \\
\hline After $\mathrm{PCl}$ & $22.38 \pm 3.07$ & $6.23 \pm 0.87$ & $1.25 \pm 0.18$ & $71.93 \pm 5.06$ \\
\hline \multicolumn{5}{|l|}{$t / P$} \\
\hline Between 2 groups before $\mathrm{PCl}$ & $1.190 / 0.235$ & $0.085 / 0.933$ & $0.150 / 0.881$ & $0.509 / 0.611$ \\
\hline Between 2 groups after $\mathrm{PCl}$ & $16.594 / 0.000$ & $27.088 / 0.000$ & $29.283 / 0.000$ & $33.241 / 0.000$ \\
\hline Control group & $30.889 / 0.000$ & $64.774 / 0.000$ & $69.159 / 0.000$ & $41.761 / 0.000$ \\
\hline
\end{tabular}

Data are mean \pm SD unless noted otherwise. AST, aspartate aminotransferase; BNP, brain natriuretic peptide; CK-MB, creatine kinase-MB; cTnT, cardiac troponin T; $\mathrm{PCl}$, percutaneous coronary intervention;

after the infarct-related artery was identified by coronary angiography. Based on conventional drug therapy, levosimendan (5 mL: $12.5 \mathrm{mg}$; NMPN H20100043, Chengdu SNBioPharm Co.) was given $30 \mathrm{~min}$ before PCI in the observation group. It was intravenously pumped at a loading dose of $12 \mu \mathrm{g} / \mathrm{kg}$ for 10 to $15 \mathrm{~min}$, and then intravenously infused at a rate of 0.1 $\mu \mathrm{g} \cdot \mathrm{kg}^{-1} \cdot \mathrm{min}^{-1}$. If the patient could tolerate, the infusion rate was increased to 0.15 to $0.2 \mu \mathrm{g} \cdot \mathrm{kg}^{-1} \cdot \mathrm{min}^{-1}$, and the infusion continued for $24 \mathrm{~h}$. In the control group, dobutamine $(2 \mathrm{~mL}$ : 20 mg; NMPN H33020471, Zhejiang Ruixin Pharmaceutical Co.) was intravenously injected at an initial rate of $2 \mu \mathrm{g} \cdot \mathrm{kg}^{-1}$ . $\mathrm{min}^{-1}$. Then the infusion rate was increased to $4 \mu \mathrm{gg} \cdot \mathrm{kg}^{-1}$. $\mathrm{min}^{-1}$, and the infusion continued for $24 \mathrm{~h}$. After operation, the patients were given general supportive treatments such as bed rest, electrocardiogram monitoring, and urethral catheterization and routine treatments such as lipid regulation, blood pressure reduction, vascular dilation, heart rate reduction, and antiplatelet and anticoagulation therapy.

\section{Collection of Baseline Data}

Sex, age, smoking history, diabetes, hypertension, hyperlipidemia, infarct-related arteries, number of diseased blood vessels, duration from onset to consultation, door-to-balloon time, intraoperative dosage of contrast agent, and duration of hospital stay were recorded in both groups.

\section{Thrombolysis in Myocardial Infarction (TIMI) Grading}

Before and after PCI, TIMI grading was conducted: grade 0 , no contrast agent passed through the stenotic artery, and no blood perfusion was seen; grade 1, a small amount of contrast agent passed through the stenotic artery, the distal blood vessel had no filling, and mild perfusion was seen; grade 2, the contrast agent obviously passed through the stenotic artery, the distal blood vessel could be filled but the blood flow was slow, and partial perfusion was seen; and grade 3, the contrast agent obviously passed through the stenotic artery, the distal blood vessel could be quickly filled, and total perfusion was observed.

\section{Detection of Myocardial Injury Markers}

Before treatment and $72 \mathrm{~h}$ after treatment, peripheral venous blood was drawn from patients. Plasma aspartate aminotransferase (AST), creatine kinase-MB (CK-MB), cardiac troponin $\mathrm{T}(\mathrm{cTnT})$, and brain natriuretic peptide (BNP) were detected by enzyme-linked immunosorbent assay.

\section{Detection of LVR Indices}

Before treatment and 3 months after treatment, left ventricular end-systolic diameter (LVESD), left ventricular end-diastolic diameter (LVEDD), and left ventricular enddiastolic volume (LVEDV) were measured using the LOGIQE portable Doppler ultrasound diagnostic apparatus and Vivid E9 color Doppler ultrasound diagnostic apparatus (GE), and LVEF was calculated.

\section{Follow-Up}

All patients were followed up by outpatient clinic for $>6$ months. In addition to related examinations, the development of LVR was mainly observed. LVR was defined as an increase in LVEDV at 3 months of follow-up by $\geq 20 \%$ compared with that before PCI.

\section{Statistical Analysis}

SPSS 16.0 software was used for statistical analysis. Numerical data were expressed as $\mathrm{n}(\%)$, and $\chi^{2}$ test was performed for intergroup comparison. Measurement data were expressed as (mean \pm standard deviation $[\mathrm{SD}]$ ), and independent-samples $t$ test was performed for intergroup comparison. First, repeated-measures analysis of variance was used to analyze the difference between 2 groups and the 
Table 4. LVR indices

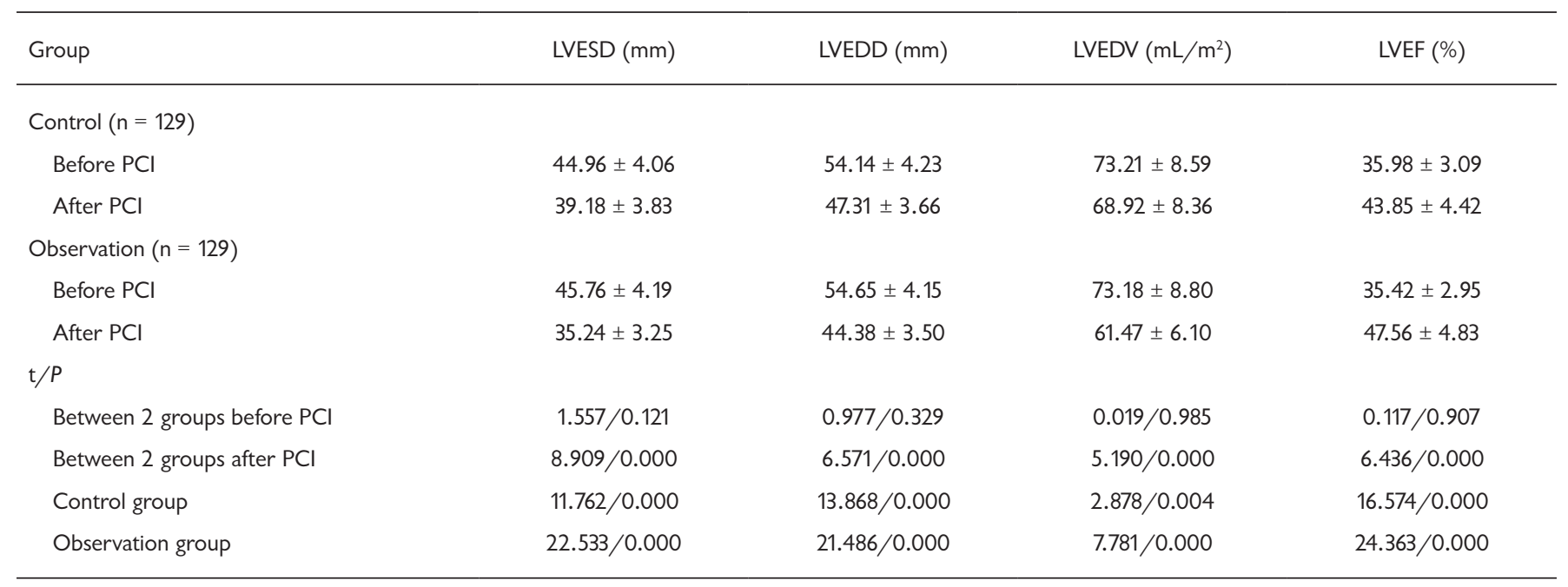

Data are mean \pm SD unless noted otherwise. LVEDD, left ventricular end-diastolic diameter; LVEDV, left ventricular end-diastolic volume; LVEF, left ventricular ejection fraction; LVESD, left ventricular end-systolic diameter; LVR, left ventricular remodeling; PCI, percutaneous coronary intervention.

time difference in each measured value. If there was a difference, least significant difference $t$ test was further adopted to compare the intergroup difference at each time point, and Student-Newman-Keuls q test to compare the time difference in each group. The influencing factors for LVR after myocardial reperfusion in AMI patients undergoing PCI were analyzed using the binary logistic regression model. $\alpha=0.05$ was set as the test level, and 2 -tailed $P<.05$ suggested a statistically significant difference.

\section{RESULTS}

\section{Baseline Clinical Data}

There were no statistically significant differences in the number of male cases, age, smoking history, diabetes, hypertension, hyperlipidemia, infarct-related arteries, number of diseased blood vessels, duration from onset to consultation, door-to-balloon time, intraoperative dosage of contrast agent, or duration of hospital stay between the control and observation groups $(P>.05)$ (Table 1$)$.

\section{TIMI Grades}

There was no statistically significant difference in the TIMI grade between the control and observation groups before and after PCI $(P>.05)$. After treatment, varying degrees of blood perfusion were found, and the TIMI grade was better than that before treatment in both groups $(P<.05)$ (Table 2$)$.

\section{Myocardial Injury Markers}

Before treatment, no statistically significant differences were found in AST, CK-MB, cTnT, or BNP between the 2 groups $(P>.05)$. After treatment, the levels of AST, CK-MB, cTnT, and BNP all declined in both groups, more significantly in the observation group $(P<.05)$ (Table 3$)$.

\section{LVR Indices}

Before treatment, LVESD, LVEDD, LVEDV, and LVEF had no statistically significant differences between the 2 groups $(P>.05)$. After treatment, LVESD, LVEDD, and LVEDV all declined and LVEF rose in both groups, more significantly in the observation group $(P<.05)$ (Table 4$)$.

\section{Univariate Analysis Results of LVR and Non-LVR Groups}

According to the definition of LVR, the cases were divided into LVR ( $\mathrm{n}=37)$ and non-LVR $(\mathrm{n}=221)$ groups. The number of male patients, age, smoking history, diabetes, hypertension, hyperlipidemia, infarct-related arteries, number of diseased blood vessels, duration from onset to consultation, door-toballoon time, intraoperative dosage of contrast agent, duration of hospital stay, LVESD, LVEDD, LVEDV, and LVEF had no statistically significant differences between the 2 groups $(P>.05)$. In the LVR group, the patients were older; the levels of AST, CK-MB, cTnT, and BNP were higher; and there were fewer cases of levosimendan preconditioning, with statistically significant differences versus the non-LVR group $(P>.05)$ (Table 5).

\section{Binary Logistic Regression Analysis Results of LVR after Myocardial Reperfusion in AMI Patients Undergoing PCI}

The variables with statistically significant differences in the LVR and non-LVR groups were used as independent variables; the actual measured values of age, AST, CK-MB, cTnT, and BNP were taken; and levosimendan preconditioning was assigned values (no $=1$, yes $=0$ ). The occurrence of LVR at 3 months of follow-up was used as the dependent variable, and assigned values (LVR $=1$, non-LVR $=0)$. The above factors were incorporated into the binary logistic regression model, and the following equation was obtained: $\operatorname{logit}(\mathrm{P})=-37.894+0.105 \times$ age $+0.077 \times \mathrm{BNP}$ $-3.953 \times$ levosimendan preconditioning. It was found that 
Table 5. Univariate analysis results of LVR and non-LVR groups

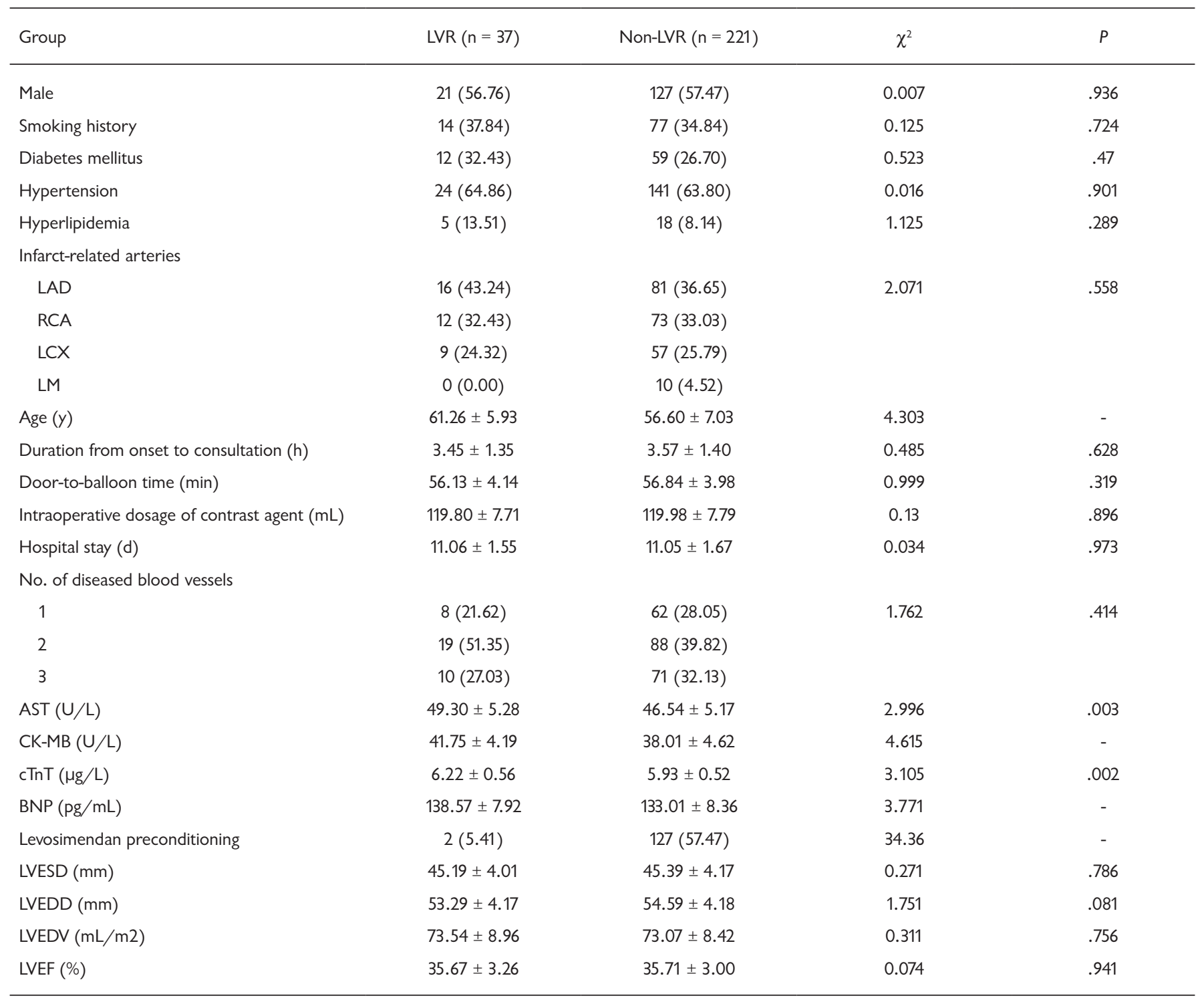

Data are $\mathrm{n}(\%)$ or mean \pm SD. AST, aspartate aminotransferase; BNP, brain natriuretic peptide; CK-MB, creatine kinase-MB; cTnT, cardiac troponin T; LAD, left anterior descending; LCX, left circumflex branch; LM, left main coronary artery; LVEDD, left ventricular end-diastolic diameter; LVEDV, left ventricular enddiastolic volume; LVEF, left ventricular ejection fraction; LVESD, left ventricular end-systolic diameter; LVR, left ventricular remodeling; RCA, right coronary artery.

age and BNP were risk factors for LVR after myocardial reperfusion in AMI patients undergoing PCI, whereas levosimendan preconditioning was a protective factor $(P<.05)$. The risk of LVR after myocardial reperfusion in 1-yearolder AMI patients undergoing PCI was 1.111 times that in 1-year-younger patients, such that a risk in patients with BNP increased by $1 \mathrm{pg} / \mathrm{mL}$ was 1.080 times that in patients with BNP decreased by $1 \mathrm{pg} / \mathrm{mL}$, and such that a risk in patients receiving levosimendan preconditioning was 0.019 times that in patients not receiving levosimendan preconditioning (Table 6).

\section{DISCUSSION}

PCI, which has been widely applied in the treatment of AMI patients, can promptly recanalize the occluded blood vessels, restore the blood circulation in coronary artery, and reduce the irreversible necrosis of myocardial cells, thereby effectively reducing the infarction area. Although blood perfusion can be restored in long-term ischemic tissues with the sudden recanalization of the coronary artery, varying degrees of damage will be caused to vascular endothelial cells and myocardial cells in the reperfusion region [Choi 2019]. In the 
Table 6. Binary logistic regression analysis results of LVR after myocardial reperfusion in AMI patients undergoing PCI

\begin{tabular}{|c|c|c|c|c|c|c|c|}
\hline BNP & 0.077 & 0.029 & 7.173 & 0.007 & 1.080 & 1.021 & 1.143 \\
\hline Levosimendan preconditioning & -3.953 & 0.856 & 21.323 & 0.000 & 0.019 & 0.004 & 0.103 \\
\hline
\end{tabular}

AMI, acute myocardial infarction; BNP, brain natriuretic peptide; $\mathrm{Cl}$, confidence interval; $\mathrm{PCl}$, percutaneous coronary intervention.

present study, different degrees of blood perfusion could be observed in both groups after PCI, and the TIMI grade was greatly improved, further confirming the effectiveness of PCI in the treatment of AMI.

AST, CK-MB, and cTnT jointly form the myocardial enzyme spectrum and are used to assess myocardial injury in patients. BNP synthesized by myocardial cells is a natural hormone able to regulate cardiac function. It has been shown that the calcium-sensitizing effect of levosimendan is achieved by direct binding to troponin [Lin 2018]. Recombinant human BNP obviously reduces the levels of blood AST and CK-MB after reperfusion in patients with acute ST segment-elevation MI, indicating that it can protect cardiac function [Li 2020]. Both recombinant human BNP and levosimendan can lower AST levels in AMI patients undergoing emergency PCI, but the effect of the former was greater [Kreusser 2019]. In this study, levosimendan preconditioning could markedly reduce the levels of blood AST, CK-MB, cTnT, and BNP in AMI patients after PCI, consistent with the above reports. It can be seen that levosimendan conditioning before PCI can protect cardiac function in AMI patients.

Clinically, LVR is mainly manifested as cardiomyocyte hypertrophy, atrophy, apoptosis, necrosis, and interstitial fibrosis, which is the key to the transition of cardiac function from a compensated state to a decompensated state and also the pathological basis of the development of cardiovascular disease to heart failure. It has previously been reported that a levosimendan treatment group had higher stroke volume and LVEF but lower BNP levels than a dobutamine group after operation, indicating that levosimendan is better in improving the cardiac function of AMI patients [Meng 2016]. In addition, levosimendan is far superior to milrinone in improving LVESD, LVEDD, and LVEF [Faisal 2019]. Moreover, levosimendan can effectively improve left heart function, relieve ventricular hypertrophy, and promote the recovery of ventricular structure in elderly patients with ischemic cardiomyopathy and heart failure [Kirlidis 2009]. Similarly, the results in this study revealed that levosimendan conditioning before PCI could promote the recovery of left ventricular structure in AMI patients, consistent with the above research reports. The possible reason is that levosimendan can dilate blood vessels to improve hemodynamics and reduce microcirculation resistance and cardiac load, thereby protecting the myocardium. In addition, levosimendan is able to regulate cytokines and neuroendocrine and antagonize the oxidative stress response, thereby reducing myocardial apoptosis and repairing damaged myocardial cells.

Although the detailed mechanism of ventricular remodeling after AMI remains unclear, it is believed that genetic factors, mechanical factors, and the neuro-endocrine system are all closely related to the occurrence of ventricular remodeling [Almeida 2020]. Age, course of disease, left atrial diameter, LVEDD, and LVEDD are influencing factors for left ventricular reverse remodeling in patients with atrial fibrillation accompanied by left ventricular hypertrophy [van Diepen 2020]. Lan et al [2019] analyzed the correlation between the thyroid-stimulating hormone level and LVR in ST segmentelevation AMI and found that the baseline thyroid-stimulating hormone $<1.38 \mathrm{mU} / \mathrm{L}$, peak level of high-sensitivity $\mathrm{c} T \mathrm{n} \mathrm{T}$, and microvascular obstruction were independent risk factors for LVR.

In this study, the influencing factors included in the analysis were different from those in the above reports. The results showed that age and BNP were risk factors for LVR after myocardial reperfusion in AMI patients undergoing PCI, whereas levosimendan preconditioning was a protective factor $(P<.05)$. The reason is that LVR hardly occurs in young patients owing to the low longitudinal strain of the left ventricle, BNP can be synthesized and secreted by atrial or ventricular myocytes, and levosimendan preconditioning can protect cardiac function and facilitate the recovery of left ventricular structure.

This study had deficiencies. For example, the sample size was not large enough, it was a single-center study, and the influencing factors included in the binary logistic regression model were not comprehensive enough, so the experimental results may be biased. In the future, the sample size will be expanded in multicenter studies, and more influencing factors will be collected to further confirm the conclusion in this study and provide a more detailed and reliable theoretical basis for clinical treatment.

\section{Conclusions}

Levosimendan preconditioning can protect cardiac function and promote the recovery of left ventricular structure. Age and BNP are risk factors for LVR after myocardial reperfusion in AMI patients undergoing PCI, and levosimendan preconditioning is a protective factor. 


\section{REFERENCES}

Agostoni P, Farmakis DT, García-Pinilla JM, et al. Haemodynamic balance in acute and advanced heart failure: An expert perspective on the role of levosimendan. Card Fail Rev 2019;5:155-161.

Almeida RCM, Jorge AJL, Rosa MLG, et al. Left ventricular remodeling patterns in primary healthcare. Arq Bras Cardiol 2020;114:59-65.

Chinese Society of Cardiology of Chinese Medical Association, Editorial Board of Chinese Journal of Cardiology. Guidelines for diagnosis and treatment of acute ST-segment elevation myocardial infarction [in Chinese]. Chin J Cardiol 2015;43:380-393.

Choi KH, Song YB, Lee JM, et al. Impact of intravascular ultrasoundguided percutaneous coronary intervention on long-term clinical outcomes in patients undergoing complex procedures. JACC Cardiovasc Interv 2019;12:607-620.

Elliott J, Kelly SE, Bai Z, et al. Optimal duration of dual antiplatelet therapy following percutaneous coronary intervention: Protocol for an umbrella review. BMJ Open 2017;7:e015421.

Faisal SA, Apatov DA, Ramakrishna H, Weiner MM. Levosimendan in cardiac surgery: Evaluating the evidence. J Cardiothorac Vasc Anesth 2019;33:1146-1158.

Kirlidis TT, Skoularigis J, Tsaknakis KT, Karayiannis G, Tsaknakis TK, Triposkiadis F. The influence of beta-blockade on the hemodynamic effects of levosimendan in elderly (>or= 70 years) patients with acutely decompensated systolic heart failure. Int J Clin Pharmacol Ther 2009;47:454-459.
Kreusser MM, Raake PW. Inpatient Therapeutic Options. In: Feldman D, Mohacsi P, eds. Heart Failure. Cardiovascular Medicine. Springer, Cham, Switzerland; 2019; 165-170.

Lan J, Gao GL, Zhang Y. Correlation between thyroid-stimulating hormone levels and left ventricular remodeling in patients with ST-segment elevation myocardial infarction [in Chinese]. Prevent Treat CardioCereb-Vasc Dis 2019;19:497-500.

Li N, Gao X, Wang W, Wang P, Zhu B. Protective effects of recombinant human brain natriuretic peptide on the myocardial injury induced by acute carbon monoxide poisoning. Cardiovasc Diagn Ther 2020;10:1785-1794.

Lin YK, Chen YC, Chen YA, Huang JH, Chen SA, Chen YJ. Levosimendan differentially modulates electrophysiological activities of sinoatrial nodes, pulmonary veins, and the left and right atria. J Cardiovasc Electrophysiol 2018;29:1150-1158.

Mehta RH, Leimberger JD, van Diepen S, et al. Levosimendan in patients with left ventricular dysfunction undergoing cardiac surgery. $\mathrm{N}$ Engl J Med 2017;376:2032-2042.

Meng JB, Hu MH, Lai ZZ, et al. Levosimendan versus dobutamine in myocardial injury patients with septic shock: A randomized controlled trial. Med Sci Monit 2016;22:1486-1496.

van Diepen S, Mehta RH, Leimberger JD, et al. Levosimendan in patients with reduced left ventricular function undergoing isolated coronary or valve surgery. J Thorac Cardiovasc Surg 2020;159:2302-2309.e6.

Zhou S, Zhang L, Li J. Effect of levosimendan in patients with acute decompensated heart failure: A meta-analysis. Herz 2019;44:630-636. 